\title{
A Recurrent Theme: A Nationwide Analysis of Hospitalization for Cyclic Vomiting Syndrome
}

\author{
Robert Issenman ${ }^{1,2,3}$
}

Published online: 13 February 2017

(c) Springer Science+Business Media New York 2017

In the past 25 years, a resurgence of interest in the cyclic vomiting syndrome (CVS) has occurred largely due to the efforts by the North American Cyclic Vomiting Syndrome Association (CVSA) to raise awareness of the syndrome [1]. First described in 1891, the condition is comprised of bouts of recurrent vomiting accompanied by intense nausea in which the patient returns to original state of health between episodes. While symptoms can vary considerably among a group of patients, episodes are usually stereotypical for individuals. Despite the availability of extensive diagnostic procedures, identification of the problem is often delayed since CVS is a diagnosis of exclusion; furthermore, development of effective treatment paradigms can be elusive.

Compared with other GI disorders, CVS is a relatively uncommon condition for which hospitalization occurs only in a subset of patients. Nevertheless, by definition, symptoms can reoccur, accounting for considerable disruption to the quality-of-life of the patient and their families. CVS is thought to occur in as many as $1 \%$ of children; at diagnosis, children report having 4-12 episodes/year, often occurring during major holidays and family vacations. The presence of this syndrome in adult populations has only been recently appreciated [2]. The series of adult 41 patients provided by Dr. David Fleisher in a 1994 publication catalogued the considerable health and social burden

Robert Issenman

issenman@mcmaster.ca

1 Departments of Pediatrics and Emergency Medicine, McMaster Children's Hospital, Hamilton, ON, Canada

2 McMaster University, Hamilton, ON, Canada

3 Centre for Child and Youth Digestive Health, Hamilton, ON, Canada experienced by adults with CVS [3]. Among the 50\% of most severely affected patients, a subset (7) had been treated in emergency departments (EDs) more than 100 times each. In a different population survey of CVS patients and families, failure to diagnose CVS occurred in $93 \%$ of ED visits.

From the perspective of a GI clinician, Hejazi and McCallum [4] indicated that 3\% of patients seen in a large adult GI motility referral center were investigated for CVS. Another adult motility program reported the syndrome in $14 \%$ of their patients. Although physicians and hospitals have sought targeted solutions for "low prevalence high intensity" patients such as these, it has proven difficult to develop a sense of the scope of the problem outside of subspecialty clinics in academic centers.

Self-reported information of the impact on individual patients has been gleaned from a national survey sponsored by the CVSA [5]. This survey and case series provide only a partial picture of the patient experience. Until the development of International Classification of Diseases, Tenth Edition (ICD-10), CVS lacked a specific diagnostic code, complicating the assessment of its impact on US hospital healthcare utilization.

The paper by Bhandari and Venkatesan [6] published in this issue of Digestive Diseases and Sciences describes patients hospitalized for CVS using data obtained from a national administrative database. These authors searched the Nationwide Inpatient Sample database for a 2-year period between January 2010 and December 2011 in order to detect the frequency and intensity of hospital encounters. Using the ICD search term for chronic vomiting, they identified 20,952 hospitalizations that were compared with a comparison sample of over 40,000 hospitalizations. While morbidity was low, hospitalizations for chronic vomiting resulted in numerous, often-repeated 
investigations with overall costs estimated to be $\$ 400$ million over 2 years.

A number of subthemes were explored. In most populations, CVS overlaps with migraine, with a positive family history of migraine in up to $82 \%$ of children and $24-70 \%$ of adults. Bhandari reported that patients hospitalized with chronic vomiting were significantly more likely to have a concurrent diagnosis of migraine headache. Although it is not surprising that anxiety and depression were more common in these patients hospitalized with a chronic relapsing health issue, it is known from conditioning experiments that anticipatory anxiety itself can amplify the severity and frequency of vomiting. While dysautonomia is uncommon overall, it is more common in these CVS patients than in controls, supporting clinical studies suggesting that in some patients a degree of dysautonomia is linked to the underlying pathophysiology of the condition.

A final theme relates to the contribution of drugs toward disease pathogenesis. Although these patients were no more likely to report the use of narcotics, the prevalence of marijuana use was much higher among the patients in this study. Non-medical marijuana use references the oft-reported pattern of CVS in young patients who also resort to frequent and extended use of hot showers [7, 8]. While the mechanism is not understood, some patients find that standing in a hot shower to be the only relief for the intense nausea characteristic of CVS. Physiologists have reported that endocannabinoid receptors are downregulated through prolonged tetrahydrocannabinol (THC) exposure and in case series [9].

CVS has resolved in patients convinced to abstain from use of this drug. In view of the current trend to legalization of marijuana, it is easy to predict a substantial increase in the numbers of CVS-related ED presentations. One publication suggested a doubling of CVS-related ED visits following cannabis legalization in Colorado [10]. By contrast, the recognition of CVS in a subset of patients seemingly reliant on marijuana can easily lead to demonization rather than a serious attempt at diagnosis and development of an effective medical plan for the patient's care.

In view of the frequency of occurrence and the burden of disease revealed in this paper, it is worth contemplating the interventions that are likely to be most effective in meeting the relatively high needs of a small number of patients. At present, a working group of the ANMS has been struck to develop evidence-based guidelines for the recognition and management of CVS in adults, with the goal of providing practitioners with tools to more successfully care for patients. Even a cursory literature review suggests that little Level 1 evidence exists to support the expected recommendations. Regardless of the absence of randomized controlled trials, there is considerable opportunity to improve the diagnostic accuracy and treatment options for this patient population. Chief among these is a strategy to provide patients with a confident CVS diagnosis in order to avoid the pattern of unnecessary investigations, erroneous diagnosis, and mischaracterization as drug seekers. As the gateway to hospitalization, improved ED management would help professionals and patients alike. Expeditious triage and institution of intravenous hydration, sedation, and anti-emesis would likely be most effective, as has been the case for the treatment of migraine headache.

While CVS is not solely the purview of gastroenterologists, many GI clinicians will find themselves caring for these patients. The overarching goal should be to prevent hospitalization and social and occupational disruption. The physicians most likely to succeed are those clinicians who meet with patients when they are well in order to develop a treatment plan for the patient's use when they become sick.

There are many models that can be adapted to CVS care. Disease self-management is a well-accepted concept for patients with asthma and diabetes. These treatment plans often employ the early institution of home medication and a step up approach to match the severity of the attack. As in migraine, early recognition and institution of treatment will abort attacks for most patients. For the patients who escalate quickly to debilitating nausea and vomiting, an early visit to an ED or outpatient infusion center is optimal, akin to the concept of the "golden hour," a well-established concept in the recognition and treatment of stroke and acute cardiac events. Although CVS may not be lifethreatening, improved efficiency in dealing with CVS patients provides superior care for the individual patient, diverting resources to other seriously ill patients in the ED.

In summary, in CVS there is a sense that in the clinic and hospital environments, the lack of expeditious care amplifies symptoms through the mechanism of anticipatory nausea. The most promising intervention to improve care for these patients is likely to involve improving the processes used by the EDs in treating symptoms that are recurrent and stereotypical. These process improvements may prove more important than the specifics of the treatments provided.

\section{References}

1. Li BUK, Issenman R, Sarna SK. Proceedings of the 2nd International Symposium on Cyclic Vomiting Syndrome. Dig Dis Sci. 1999;44:1S-120S.

2. Abell TL, Adams KA, Boles RG, Chong SKF, Fleisher DR, et al. Cyclic vomiting syndrome in adults. Neurogastroenterol Motil. 2008;20:269-284.

3. Fleisher D, Gornowicz B, Adams K, Feldman E. Cyclic vomiting syndrome in 41 adults: the illness, the patients and the problems of management. BioMed Central Med. 2005;3:20-28. 
4. Hejazi RA, McCallum RW. Review article: cyclic vomiting in adults. Rediscovering and redefining an old entity. Aliment Pharmacol Ther. 2011;34:263-273.

5. Venkatesan T, Tarbell S, Adams K, et al. A survey of E, Department Use in patients with cyclic vomiting syndrome. BMC Emerg Med. 2010;10:4.

6. Bhandhari S, Venkatesan T. Clinical characteristics, co-morbidities and hospital outcomes in hospitalizations in cyclic vomiting syndrome: a nationwide analysis. Dig Dis Sci. (Epub ahead of print). doi:10.1007/s10620-016-4432-7.

7. Wallace EA, Andrews SE, Garmany CL, et al. Cannabinoid hyperemesis syndrome: literature review and proposed diagnosis and treatment algorithm. South Med J. 2011;104:659-664.
8. Mohammad F, Panchoo K, Barthalomew M, Maharaj D. Compulsive showering and cannabinoid use. Am $J$ Case Rep. 2013;14:326-328.

9. Venkatesan T, Zadvornova Y, Raff H, et al. Endocannabinoidrelated lipids are increased during an episode of cyclic vomiting syndrome. Neurogastroenterol Motil. 2016;28:1409-1418.

10. California Medical Association Marijuana Technical Advisory Committee. Cannabis and the Regulatory Void: Background Paper and Recommendations. http://www.cmanet.org/files/pdf/ news/cma-cannabis-tac-white-paper-101411.pdf. 